B. Abdellaoui · I. Peral Alonso

\title{
A note on a critical problem with natural growth in the gradient
}

To Antonio Ambrosetti, master and friend, on the occasion of his 60th birthday

Received August 21, 2005 and in revised form December 11, 2005

Abstract. The paper analyzes the influence on the meaning of natural growth in the gradient of a perturbation by a Hardy potential in some elliptic equations. Indeed, in the case of the Laplacian the natural problem becomes

$$
-\Delta u-\Lambda_{N} \frac{u}{|x|^{2}}=\left|\nabla u+\frac{N-2}{2} \frac{u}{|x|^{2}} x\right|^{2}|x|^{(N-2) / 2}+\lambda f(x) \text { in } \Omega, \quad u=0 \text { on } \partial \Omega,
$$

$\Lambda_{N}=((N-2) / 2)^{2}$. This problem is a particular case of problem 2 . Notice that $(N-2) / 2$ is optimal as coefficient and exponent on the right hand side.

Keywords. Elliptic equations, Hardy potential, quadratic growth in the gradient, optimal summability

\section{Introduction and preliminaries}

The problem

$$
-\Delta u=|\nabla u|^{2}+f(x), \quad u \in W_{0}^{1,2}(\Omega),
$$

where $\Omega$ is a bounded domain in $\mathbb{R}^{N}$, appears in applications as the stationary form of several mathematical models. Indeed, significant examples are the viscosity approximation of Hamilton-Jacobi type equations from stochastic control theory (see [15]), the physical theory of growth and roughening of surfaces, where it is known as the KardarParisi-Zhang equation (see [14]), and some models of propagation of flames (see [5]). It is worthwhile to note that the classical change of variable $v=e^{u}-1$ connects problem (1) with the study of positive solutions to Schrödinger equations. We refer to the recent paper [16] for an updated list of references about this interesting problem but collateral with the purpose of this work. Equations of the form (1) have been widely studied in the literature: see for example [1], [6], [7], [12] and the references therein. In [1] a deep relation between problem (1) and some linear elliptic problem with measure data is clarified and a

Research partially supported by Project MTM2004-02223 of M.E.C. Spain.

B. Abdellaoui, I. Peral Alonso: Departamento de Matemáticas, Universidad Autónoma de Madrid, 28049 Madrid, Spain; e-mail: boumediene.abdellaoui@uam.es, ireneo.peral@uam.es

Mathematics Subject Classification (2000): 35D10, 35J20, 35J25, 35J70, 46E30 
complete classification of the solutions in correspondence to the family of singular measures concentrated on a set of zero capacity is obtained. The presence of the critical zero order term $u|x|^{-2}$ motivates the nonexistence result for $-\Delta u-c u /|x|^{2}=|\nabla u|^{2}+f(x)$, $c>0, u \in W_{0}^{1,2}(\Omega)$ (as is easy to check by using some nonexistence results in [2]).

In this paper we will analyze the meaning of the natural growth in the gradient under the presence of a critical potential. Precisely we will study the following elliptic problem:

$$
\left\{\begin{array}{l}
-\operatorname{div}\left(|x|^{-2 \gamma} \nabla u\right)-\Lambda_{N, \gamma} \frac{u}{|x|^{2(\gamma+1)}}=\frac{\left|\nabla u+\frac{N-2(\gamma+1)}{2} \frac{u}{|x|^{2}} x\right|^{2}}{|x|^{2 \gamma-\beta}}+\lambda f(x) \text { in } \Omega, \\
u=0 \text { on } \partial \Omega,
\end{array}\right.
$$

where $\Omega$ is a bounded open set in $\mathbb{R}^{N}, N \geq 3$, such that $0 \in \Omega, \gamma<(N-2) / 2, \lambda$ is a positive constant, $f(x)$ is a positive measurable function, $\Lambda_{N, \gamma}=(N-2(\gamma+1))^{2} / 4$ and $\beta \geq(N-2(\gamma+1)) / 2$ (see Theorem 3.1 below to motivate the condition on $\beta$ ). We point out that if $\Lambda_{N, \gamma}$ is replaced by $0<c<\Lambda_{N, \gamma}$, similar results could be obtained; for brevity we only handle the critical case. The term $u(x)|x|^{-2(\gamma+1)}$ is related to the following weighted Hardy-Sobolev inequality (see for instance [2]):

$$
\Lambda_{N, \gamma} \int_{\Omega} u^{2}(x)|x|^{-2(\gamma+1)} d x \leq \int_{\Omega}|\nabla u|^{2}|x|^{-2 \gamma} d x \quad \text { for all } u \in \mathcal{C}_{0}^{\infty}(\Omega)
$$

where the optimal constant, $\Lambda_{N, \gamma}$, is not achieved. It is worth pointing out that the right hand side in (2) is suggested by the improved inequalities in [3] and [17].

The paper is organized as follows. We start by studying problems of the form

$$
\left\{\begin{array}{l}
\mathcal{L}_{N, \gamma}(u) \equiv-\operatorname{div}\left(|x|^{-2 \gamma} \nabla u\right)-\Lambda_{N, \gamma} u(x)|x|^{-2(\gamma+1)}=g \quad \text { in } \Omega, \\
u=0 \text { on } \partial \Omega,
\end{array}\right.
$$

where $g$ is an integrable function with respect to some weight, which will be precisely determined. The presence of the term $u(x)|x|^{-2(\gamma+1)}$ implies that there is no existence result for $g \in L^{1}(\Omega)$, or more generally for a bounded Radon measure $\mu$ as a second member in (4). In this direction we will obtain sufficient conditions to have existence of a solution in a weaker sense. Such conditions in the case of an integrable function are also necessary. In Section 3 we study the elliptic problem with critical growth in the gradient understood as the right hand side in problem 2. In Subsection 3.1 we give a general nonexistence result according to the weight, which motivates the hypotheses on $\beta$ and the structure of the right hand side. Moreover, we prove that $(N-2(\gamma+1)) / 2$ is optimal as coefficient and as exponent on the right hand side of problem (2). Subsection 3.2 deals with a semilinear problem connected to the main equation, furthermore, we obtain existence and nonexistence results with optimal regularity. The regularity of general solutions to (2) is analyzed in Subsection 3.3. This regularity result is the starting point to connect the study of nonuniqueness in problem (2) with some linear problems with measure data, and to obtain multiplicity of solutions. Notice that the results are new even in the case $\gamma=0$, corresponding to the Laplacian and the classical Hardy potential. We point out that the results require the use of a natural energy space $H_{\gamma}$ which is defined below. 


\subsection{Functional setting}

We define the space $H_{\gamma}$ as the completion of $\mathcal{C}_{0}^{\infty}(\Omega)$ endowed with the norm

$$
\|\phi\|_{H_{\gamma}}^{2}=\int_{\Omega}|\nabla \phi|^{2}|x|^{-2 \gamma} d x-\Lambda_{N, 2, \gamma} \int_{\Omega} \phi^{2}(x)|x|^{-2(\gamma+1)} d x .
$$

It is not difficult to see that $H_{\gamma}$ is a Hilbert space and that the norm of $H_{\gamma}$ can be written in the form

$$
\|\phi\|_{H_{\gamma}}^{2}=\left.\left.\int_{\Omega}|x|^{-2 \gamma}\left|\nabla \phi+\frac{N-2(\gamma+1)}{2} \phi(x)\right| x\right|^{-2} x\right|^{2} d x .
$$

The space $H_{\gamma}$ appears in a natural way as a consequence of the improved Hardy-Sobolev inequalities (see [3] and [17] and the references therein). It is the natural energy space to study problem (2) and, moreover, motivates the right hand side in (4). We will use the notation $L_{\theta}^{q}(\Omega) \equiv L^{q}\left(|x|^{-\theta} d x, \Omega\right)$. We also define the weighted Sobolev space $\mathcal{D}_{0,(N-2) / 2}^{1,2}(\Omega)$ as the completion of $\mathcal{C}_{0}^{\infty}(\Omega)$ endowed with the norm

$$
\|\phi\|_{\mathcal{D}_{0,(N-2) / 2}^{1,2}}^{2}=\int_{\Omega}|\nabla \phi|^{2}|x|^{-(N-2)} d x .
$$

Then $\mathcal{D}_{0,(N-2) / 2}^{1,2}(\Omega)$ is also a Hilbert space. As in [10], for $u \in H_{\gamma}$, define $v(x)=$ $|x|^{(N-2(\gamma+1)) / 2} u$. It is easy to check that $v \in \mathcal{D}_{0,(N-2) / 2}^{1,2}$, and conversely, if $v \in \mathcal{D}_{0,(N-2) / 2}^{1,2}$ then $u(x)=|x|^{-(N-2(\gamma+1)) / 2} v \in H_{\gamma}$. By a direct computation we are able to prove that if $u \in H_{\gamma}$, then $T_{k}(u) \equiv u-\operatorname{sign}(u)(|u|-k)_{+} \in H_{\gamma}$ for all $k>0$ and $w=|u| /(1+|u|) \in$ $H_{\gamma}$. In this framework we have the following concept of solution: we say that $u \in H_{\gamma}$ is a weak solution to problem (2) if

$$
|x|^{\beta-2 \gamma}\left|\nabla u+\alpha \frac{u}{|x|^{2}} x\right|^{2}+f \in L^{1}(\Omega)
$$

and the equation holds in distributional sense.

\section{Right hand side belonging to $L^{1}$ or to the space of Radon measures}

In this section we consider the problem

$$
\left\{\begin{array}{l}
\mathcal{L}_{N, \gamma}(u)=v \equiv g(x)+v_{0} \quad \text { in } \Omega, \\
u=0 \text { on } \partial \Omega
\end{array}\right.
$$

where $\Omega$ is a bounded open set in $\mathbb{R}^{N}, N \geq 3,0 \in \Omega, g \in L^{1}(\Omega)$ and $v_{0}$ is a singular bounded Radon measure (singular with respect to the Lebesgue measure).

Given a Radon measure $\mu$ on $\Omega$ and a Borel set $E \subset \Omega$, we say that $\mu$ is concentrated on $E$ if for every Borel set $B, \mu(B)=\mu(B \cap E)$. If $\mu$ is concentrated on $A$ we will write $\operatorname{con}(\mu)=A$. We begin with the following definition. 
Definition 2.1. We say that $u$ is a weak solution of problem $(5)$ if $u, u|x|^{-2(\gamma+1)} \in L^{1}(\Omega)$ and for all $\phi \in \mathcal{C}^{2}(\bar{\Omega})$ such that $\phi=0$ on $\partial \Omega$,

$$
\int_{\Omega} u\left(-\operatorname{div}\left(|x|^{-2 \gamma} \nabla \phi\right)-\Lambda_{N, \gamma} \phi(x)|x|^{-2(\gamma+1)}\right) d x=\langle\nu, \phi\rangle .
$$

Due to the presence of singular or degenerate coefficients and the term $u(x)|x|^{-2(\gamma+1)}$ we need to specify conditions on $g$ and $v_{0}$ to construct a weak solution. This is the result of the next theorem.

Theorem 2.2. Assume that $v=g(x)+v_{0}$ is a bounded Radon measure such that:

1. $\operatorname{con}\left(v_{0}\right) \cap B_{a}(0)=\emptyset$ where $\bar{B}_{a}(0) \subset \Omega$,

2. $|x|^{-(N-2(\gamma+1)) / 2} g \in L^{1}(\Omega)$.

Then problem (5) has a weak solution $u$ such that

(i) $T_{k}(u) \in \mathcal{D}_{0, \gamma}^{1,2}(\Omega)$,

(ii) $u \in \mathcal{M}^{p_{1}}\left(\Omega,|x|^{-2 \gamma} d x\right), p_{1}=(N-2 \gamma) /(N-2(\gamma+1))$ if $\gamma<0$ and $p_{1}=$ $N /(N-2)$ if $\gamma \geq 0\left(\mathcal{M}^{p_{1}}\left(\Omega,|x|^{-2 \gamma} d x\right)\right.$ is the corresponding Marcinkiewicz space $)$.

(iii) $|\nabla u| \in L^{q}\left(\Omega,|x|^{-2 \gamma} d x\right)$ for all $q<(N-2 \gamma) /(N-2 \gamma-1)$ if $\gamma<0, q<$ $N /(N-1)$ if $\gamma \geq 0$.

Moreover, if $v$ is a positive measure, then $u$ is positive and in this case problem (5) has a minimal weak solution.

Proof. By linearity, we can assume that the measure $v$ is positive. Consider a sequence $\left\{k_{n}\right\}$ of bounded functions such that $k_{n} \rightarrow v$ in the sense of measures. Then by the hypothesis on $v$ we can assume that $k_{n}=g_{n}+\alpha_{n}$ where $g_{n}, \alpha_{n} \in L^{\infty}(\Omega), g_{n} \uparrow g$ in $L^{1}(\Omega)$ and $|x|^{-(N-2(\gamma+1)) / 2} g_{n} \rightarrow|x|^{-(N-2(\gamma+1)) / 2} g$ in $L^{1}(\Omega), \alpha_{n} \rightarrow \nu_{0}$ in the sense of measures and $\operatorname{con}\left(\alpha_{n}\right) \subset \Omega \backslash B_{a / 2}(0)$. Hence $|x|^{-(N-2(\gamma+1)) / 2} v$ is well defined and $|x|^{-(N-2(\gamma+1)) / 2} k_{n} \rightarrow|x|^{-(N-2(\gamma+1)) / 2} v$ in the sense of measures. Let $v_{n} \in H_{\gamma}$ be the minimal solution to the problem

$$
\mathcal{L}_{N, \gamma}\left(v_{n}\right)=k_{n}, \quad v_{n \mid \partial \Omega}=0,
$$

and let $\psi_{0}$ be the unique energy solution to the problem

$$
\mathcal{L}_{N, \gamma}\left(\psi_{0}\right)=|x|^{-2(\gamma+1)}, \quad \psi_{0 \mid \partial \Omega}=0 .
$$

It is easy to check that $\psi_{0}(x) \leq c|x|^{-(N-2(\gamma+1)) / 2}$. Using $\psi_{0}$ as a test function in (7) and taking into account the hypothesis on $v$ we obtain

$$
\int_{\Omega} v_{n}(x)|x|^{-2(\gamma+1)}=\int_{\Omega} \phi_{0} k_{n} \leq C\left(\phi_{0}\right) \int_{\Omega}|x|^{-(N-2(\gamma+1)) / 2} d \nu \leq C_{1} .
$$

Thus $v_{n}(x)|x|^{-2(\gamma+1)}$ is uniformly bounded in $L^{1}(\Omega)$. By taking $T_{k}\left(v_{n}\right)$ as a test function in (7) it follows that

$$
\frac{1}{k} \int_{\Omega}|x|^{-2 \gamma}\left|\nabla T_{k}\left(v_{n}\right)\right|^{2} d x \leq C \quad \text { for all } k>0 .
$$


Hence, there exists $w_{k} \in \mathcal{D}_{0, \gamma}^{1,2}(\Omega)$ such that $T_{k}\left(v_{n}\right) \rightarrow w_{k}$ weakly in $\mathcal{D}_{0, \gamma}^{1,2}(\Omega)$ and strongly in $L_{q \gamma}^{q}(\Omega)$ for all $q<2^{*}$. Using the same argument as in [2] we conclude that $v_{n}$ is bounded in the Marcinkiewicz space $\mathcal{M}^{q_{1}}\left(\Omega, d \mu_{\gamma}\right)$ with $d \mu_{\gamma} \equiv|x|^{-2 \gamma} d x, q_{1}=$ $2_{\gamma}^{*} / 2$, where, as in [2], $2_{\gamma}^{*}=2 N /(N-2)$ if $\gamma \geq 0$ and $2_{\gamma}^{*}=2(N-2 \gamma) /(N-2(\gamma+1))$ if $\gamma<0$. Indeed, since the norm of $v_{n}$ in $\mathcal{M}^{q_{1}}\left(\Omega, d \mu_{\gamma}\right)$ is given by

$$
\left\|v_{n}\right\|_{\mathcal{M}^{q_{1}}\left(\Omega, d \mu_{\gamma}\right)}=\inf \left\{C>0: \mu_{\gamma}\left\{\left|v_{n}\right|>t\right\} \leq c t^{-q_{1}}\right\},
$$

by using estimate 9 , and the Sobolev inequality (2.7) of [2] we get

$$
\begin{aligned}
\mu_{\gamma}\left\{\left|v_{n}\right|>k\right\} & =\mu_{\gamma}\left\{\left|T_{k}\left(v_{n}\right)\right|=k\right\} \leq \int_{\Omega} \frac{\left|T_{k}\left(v_{n}\right)\right|^{2 *}}{k^{2 *}}|x|^{-2 \gamma} d x \\
& \leq C(\Omega) \frac{1}{k^{2 *}}\left(\int_{\Omega}\left|\nabla T_{k}\left(v_{n}\right)\right|^{2}|x|^{-2 \gamma} d x\right)^{2 / 2_{\gamma}^{*}} .
\end{aligned}
$$

Then we obtain $\mu\left\{x \in \Omega:\left|v_{n}\right|>k\right\} \leq C M^{2 * / 2} k^{-\left(2_{\gamma}^{*}-2 * / 2\right)}$. Hence $\left\|v_{n}\right\|_{\mathcal{M}^{q_{1}}\left(\Omega, d \mu_{\gamma}\right)} \leq C$ for all $n$. Since $L_{\gamma}^{q_{1}}(\Omega) \subset \mathcal{M}^{q_{1}}\left(\Omega, d \mu_{\gamma}\right) \subset L_{\gamma}^{q_{1}-\alpha}(\Omega)$ for all $\alpha>0$ small, we conclude that $\left\{v_{n}\right\}$ is uniformly bounded in $L_{\gamma}^{q_{1}-\alpha}(\Omega)$. In particular we get the existence of $u \in$ $L_{\gamma}^{q_{1}-\alpha}(\Omega)$ such that $v_{n} \rightarrow u$ weakly in $L_{\gamma}^{q_{1}-\alpha}(\Omega)$ and $v_{n} \rightarrow u$ a.e. in $\Omega$. Using the uniqueness of the limit we infer that $w_{k}=T_{k}(u)$ and then $T_{k}(u) \in \mathcal{D}_{0, \gamma}^{1,2}(\Omega)$ for all $k>0$. By Vitali's theorem we find that $v_{n}(x)|x|^{-2(\gamma+1)} \rightarrow u(x)|x|^{-2(\gamma+1)}$ in $L^{1}(\Omega)$. Hence $v_{n}(x)|x|^{-2(\gamma+1)}+k_{n} \rightarrow u(x)|x|^{-2(\gamma+1)}+v$ weakly in the sense of measures and then we can prove easily that $u$ is a weak solution to (5). If $v$ is a positive measure, then we can choose $k_{n} \geq 0$, thus $v_{n} \geq 0$.

The existence of a minimal solution follows by an approximation argument. Indeed, we take a sequence $\left\{g_{n}\right\}$ of bounded functions such that $g_{n} \uparrow g$ a.e. For fixed $n$ we define the sequence $\left\{v_{n, k}\right\}$ as follows. Let $v_{n, 1}$ be the solution of

$$
-\operatorname{div}\left(|x|^{-2 \gamma} \nabla v_{n, 1}\right)=g_{n}+v_{0}, \quad v_{n, 1 \mid \partial \Omega}=0,
$$

and by iteration we define $v_{n, k}$ as the solution to

$$
-\operatorname{div}\left(|x|^{-2 \gamma} \nabla v_{n, k}\right)=\Lambda_{N, \gamma} v_{n, k-1}|x|^{-2(\gamma+1)}+g_{n}+v_{0}, \quad v_{n, k \mid \partial \Omega}=0 .
$$

Then, by the comparison principle for renormalized solutions, we get $v_{n, k} \leq u$ for all $(n, k), v_{n, k} \leq v_{n, k+1}$ and $v_{n, k} \leq v_{n+1, k}$. Hence $v_{n, k} \uparrow v_{n}$ which is a solution to

$$
-\operatorname{div}\left(|x|^{-2 \gamma} \nabla v_{n}\right)=\Lambda_{N, \gamma} v_{n}|x|^{-2(\gamma+1)}+g_{n}+v_{0}, \quad v_{n \mid \partial \Omega}=0,
$$

with $v_{n} \leq u$. Notice that $v_{n} \leq v_{n+1}$. Therefore $\left\{v_{n}\right\}$ converges in $L_{\gamma}^{1}(\Omega)$ to a positive function $w$. Since $v_{n}|x|^{-2(\gamma+1)} \leq u|x|^{-2(\gamma+1)}$, we have $w|x|^{-2(\gamma+1)} \in L^{1}(\Omega)$. Thus we conclude easily that $w$ is the weak minimal solution to problem (5). Finally, by (9) and using the same ideas as in [2] (see also [4]), we conclude that $u$ satisfies the equation in the renormalized sense (see [11]).

In the case where $\nu_{0}=0$ the condition on $g$ is also necessary. This is the contents of the following result. 
Theorem 2.3. Given an integrable function $g$, problem (5) has a weak solution if and only if $|x|^{-(N-2(\gamma+1)) / 2} g \in L^{1}(\Omega)$.

Proof. The fact that the condition is sufficient is a particular case of Theorem 2.2. We prove that the condition is necessary. Without loss of generality we can assume that $g \geq 0$. If problem (5) has a weak solution, taking it as a supersolution and using an approximation argument, we see that (5) has a minimal weak solution $w$. Let $\phi_{0} \in H_{\gamma}$ be the solution to

$$
\mathcal{L}_{N, \gamma}\left(\phi_{0}\right)=1, \quad \phi_{0 \mid \partial \Omega}=0
$$

One can check that $\phi_{0}(x) \simeq c|x|^{-(N-2(\gamma+1)) / 2}$ in a neighborhood of $x_{0}=0$. Then by taking $\phi_{0}$ as a test function in problem (7) with $v_{0}=0$, and using the fact that $v_{n} \uparrow w$, we conclude that

$$
C_{1}+\int_{\Omega} w d x=\int_{\Omega} g \phi_{0} d x \geq C_{2} \int_{\Omega} g|x|^{-(N-2(\gamma+1)) / 2} d x
$$

by the monotone convergence theorem.

Remarks 2.4. (A) Notice that the weak solution $u$ is obtained as a limit of approximations (S.O.L.A.). Therefore, one can check that such a solution satisfies the equation in the renormalized sense (see [11]). Moreover if $\nu_{0} \in L^{1}(\Omega)$, then $u$ is an entropy solution in the sense of [4] (see also Theorem 4.2 in [2]).

(B) In general if $u$ is a weak positive supersolution of $\mathcal{L}_{N, \gamma}(u) \geq 0$, there exists a positive constant $c$ such that $u(x) \geq c|x|^{-(N-2(\gamma+1)) / 2}$ as $|x| \rightarrow 0$.

(C) Uniqueness for problem (5) in the framework of Definition 2.1 does not hold. For instance if $\Omega=B_{1}(0)$ is the unit ball, then $u_{0}(x)=-|x|^{-(N-2(\gamma+1))} / 2 \log (|x|)$ is a weak solution to problem $\mathcal{L}_{N, \gamma}\left(u_{0}\right)=0, u_{0 \mid \partial \Omega}=0$, and hence if $u$ is a weak solution to (5), then $u+u_{0}$ is also a solution.

(D) A weaker sense of solution could be considered (see [9]).

\section{Problem with critical growth in the gradient}

In this section we deal with the optimality of the exponent and the coefficient of the right hand side of problem 2, Consider the problem

$$
\left\{\begin{array}{l}
\mathcal{L}_{N, \gamma}(u)=|x|^{\beta-2 \gamma}\left|\nabla u+\alpha \frac{u}{|x|^{2}} x\right|^{2}+\lambda f(x), \quad u \geq 0 \quad \text { in } \Omega, \\
u=0 \text { on } \partial \Omega
\end{array}\right.
$$

where $\Omega \subset \mathbb{R}^{N}$ is a bounded domain, $N \geq 3,0 \in \Omega, \alpha \in \mathbb{R}, \beta, \lambda>0$ and $f$ is a positive function with summability that we will specify below. 


\subsection{Preliminary results: necessary conditions on $\alpha$ and $\beta$}

If (11) has a positive solution $u$, in particular it means that

$$
\left.\left.g \equiv|x|^{\beta-2 \gamma}|\nabla u+\alpha u| x\right|^{-2} x\right|^{2}+\lambda f(x) \in L^{1}(\Omega),
$$

and using the result of Theorem 2.3 we obtain

$\left.\left.\int_{\Omega}|x|^{\beta-2 \gamma}|\nabla u+\alpha u| x\right|^{-2} x\right|^{2}|x|^{-(N-2(\gamma+1)) / 2} d x<\infty, \quad \int_{\Omega} \frac{f}{|x|^{(N-2(\gamma+1)) / 2}} d x<\infty$.

Our first nonexistence result is the following; it allows us to select the admissible values of $\beta$.

Theorem 3.1. Assume $\beta<(N-2(\gamma+1)) / 2$ or $\alpha \neq(N-2(\gamma+1)) / 2$. If $f \neq 0$, then problem 111 has no solution. If $f \equiv 0$, then the unique solution is $u \equiv 0$.

Proof. Assume by contradiction that $u>0$ is a weak solution to (11). Since $\beta<$ $(N-2(\gamma+1)) / 2$ and $\gamma<(N-2) / 2$, consider $\varepsilon>0$ such that $(N-2(\gamma+1)) / 2-$ $\beta>2 \varepsilon$ and $\gamma+\varepsilon<(N-2) / 2$. Thus we obtain

$\left.\left.\int_{\Omega}|x|^{\beta-2 \gamma}|\nabla u+\alpha u| x\right|^{-2} x\right|^{2}|x|^{-(N-2(\gamma+1)) / 2} d x \geq\left.\left. C \int_{\Omega}|x|^{-2(\varepsilon+\gamma)}|\nabla u+\alpha u| x\right|^{-2} x\right|^{2} d x$.

By a direct computation,

$$
\begin{aligned}
& \int_{\Omega}|x|^{-2(\varepsilon+\gamma)}\left|\nabla \phi+\alpha \frac{\phi}{|x|^{2}} x\right|^{2} d x=\left(\frac{N-2(\varepsilon+\gamma+1)}{2}-\alpha\right)^{2} \int_{\Omega} \frac{\phi^{2}}{|x|^{2(\varepsilon+\gamma+1)}} d x \\
& +\int_{\Omega}|x|^{-2(\varepsilon+\gamma)}|\nabla \phi|^{2} d x-\left(\frac{N-2(\varepsilon+\gamma+1)}{2}\right)^{2} \int_{\Omega} \frac{\phi^{2}}{|x|^{2(\varepsilon+\gamma+1)}} d x, \quad \forall \phi \in \mathcal{C}_{0}^{\infty}(\Omega) .
\end{aligned}
$$

Consider $R>0$ such that $\Omega \subset B_{R / 2}(0)$. Then, by the improved Hardy-Sobolev inequality of [17], there exists a constant $C>0$ such that

$$
\begin{aligned}
\int_{\Omega}\left|\nabla \phi+\alpha \frac{\phi}{|x|^{2}} x\right|^{2}|x|^{-2(\varepsilon+\gamma)} d x \geq & \left(\frac{N-2(\varepsilon+\gamma+1)}{2}-\alpha\right)^{2} \int_{\Omega} \frac{\phi^{2}}{|x|^{2(\varepsilon+\gamma+1)}} d x \\
& +C \int_{\Omega} \frac{\phi^{2}}{|x|^{2(\varepsilon+\gamma+1)}}\left(\log \left(\frac{R}{|x|}\right)\right)^{-2} d x
\end{aligned}
$$

A density argument gives the same inequality for $u$. According to (B) in Remarks 2.4 $u(x) \geq c|x|^{-(N-2(\gamma+1)) / 2}$ as $|x| \rightarrow 0$, which provides a contradiction with the fact that, for all $\alpha, 12$ implies

$$
\int_{\Omega} \frac{u^{2}}{|x|^{2(\varepsilon+\gamma+1)}}\left(\log \left(\frac{R}{|x|}\right)\right)^{-2} d x<\infty .
$$

Hence $\beta=(N-2(\gamma+1)) / 2$ is optimal. 
Finally, if $\beta=(N-2(\gamma+1)) / 2$ and $\alpha \neq(N-2(\gamma+1)) / 2$, then 12 with $\varepsilon=0$ implies

$$
\int_{\Omega} \frac{u^{2}}{|x|^{2(\gamma+1)}} d x<\infty
$$

which contradicts the fact that $u(x) \geq c|x|^{-(N-2(\gamma+1)) / 2}$ as $|x| \rightarrow 0$. Hence the result follows.

Theorem 3.1 motivates why in the following we study the problem

$$
\left\{\begin{array}{l}
\mathcal{L}_{N, \gamma}(u)=|x|^{-2 \gamma}\left|\nabla u+\frac{N-2(\gamma+1)}{2} \frac{u}{|x|^{2}} x\right|^{2}|x|^{(N-2(\gamma+1)) / 2}+\lambda f(x) \quad \text { on } \Omega, \\
u=0 \text { on } \partial \Omega,
\end{array}\right.
$$

where $\lambda$ is a positive constant and $f(x)$ is a positive measurable function. Notice that problem (13) corresponds to the critical value $\beta=(N-2(\gamma+1)) / 2$.

\subsection{Existence and nonexistence}

We set $v(x)=|x|^{(N-2(\gamma+1)) / 2} u(x)$ (see [10]); then problem (13) becomes

$$
\left\{\begin{array}{l}
-\operatorname{div}\left(|x|^{-(N-2)} \nabla v\right)=|x|^{-(N-2)}|\nabla v|^{2}+\lambda|x|^{-(N-2(\gamma+1)) / 2} f(x) \text { in } \Omega, \\
v=0 \text { on } \partial \Omega .
\end{array}\right.
$$

In problem (14) we perform the classical Hopf-Cole change of dependent function, i.e. $w=e^{v}-1$. By a direct computation $w$ solves the problem

$$
\left\{\begin{array}{l}
-\operatorname{div}\left(|x|^{-(N-2)} \nabla w\right)=\lambda|x|^{-(N-2(\gamma+1)) / 2} f(x)(w+1) \quad \text { in } \Omega, \\
w=0 \text { on } \partial \Omega .
\end{array}\right.
$$

It is well known that problem 15 has a unique solution if $|x|^{-(N-2(\gamma+1)) / 2} f(x)$ is in the dual space of $\mathcal{D}_{0,(N-2) / 2}^{1,2}(\Omega)$ and $\lambda$ is small enough. For instance if $f|x|^{(N+2(\gamma-1)) / 2} \in$ $L^{p}(\Omega), p>N / 2$, the right hand side has the required regularity. Then we get the following result.

Theorem 3.2. Assume $f|x|^{(N+2(\gamma-1)) / 2} \in L^{p}(\Omega)$ and $p>N / 2$. Then for $\lambda$ small enough, there exists a unique weak solution to problem (13), $u \in H_{\gamma}$, such that

$$
e^{|x|^{(N-2(\gamma+1)) / 2} u}-1 \in \mathcal{D}_{0,(N-2) / 2}^{1,2}(\Omega) .
$$

We now give some existence and nonexistence results under some hypothesis on $f$ and the size of $\lambda$. Assume that $f$ is a measurable, nonnegative function with the following natural property (see [1] and [13]):

(A) $\exists \phi_{0} \in \mathcal{C}_{0}^{\infty}(\Omega)$ such that $\int_{\Omega} \frac{\left|\nabla \phi_{0}\right|^{2}}{|x|^{(N-2)}} d x<\lambda \int_{\Omega} f|x|^{-(N-2(\gamma+1)) / 2} \phi_{0}^{2} d x<\infty$.

As a consequence we have the following nonexistence result. 
Theorem 3.3. If $\lambda$ and $f$ satisfy hypothesis (A) above, then problem (13) has no solution.

The proof is elementary as in [1] so we omit the details.

Let $f$ be a nonnegative measurable function, and define

$$
\lambda_{1}(f)=\inf _{\phi \in \mathcal{C}_{0}^{\infty}(\Omega)} \frac{\int_{\Omega}|\nabla \phi|^{2}|x|^{-(N-2)} d x}{\int_{\Omega} f|x|^{-(N-2(\gamma+1)) / 2} \phi^{2} d x} \geq 0 .
$$

We consider the following hypothesis:

$$
f \in L^{1}\left(\Omega,|x|^{-(N-2(\gamma+1)) / 2} d x\right) \text { and } \quad \lambda_{1}(f)>0 .
$$

Theorem 3.4. If (B) holds then problem (13) has no solution in $H_{\gamma}(\Omega)$ for $\lambda>\lambda_{1}(f)$ and has a unique solution $u \in H_{\gamma}(\Omega)$ such that $e^{|x|^{(N-2(\gamma+1)) / 2} u}-1 \in \mathcal{D}_{0,(N-2) / 2}^{1,2}(\Omega)$ for $\lambda<\lambda_{1}(f)$.

Proof. If $\lambda>\lambda_{1}(f)$, then by a density argument we can show that condition (A) holds for some $\phi_{0} \in \mathcal{C}_{0}^{\infty}(\Omega)$ and therefore the nonexistence is a consequence of Theorem 3.3 . We now prove the existence result. A direct computation shows that hypothesis $(\mathbf{B})$ implies that $f \in L^{1}\left(\Omega,|x|^{-(N-2(\gamma+1)) / 2} d x\right)$ and is in the dual space of $\mathcal{D}_{0,(N-2) / 2}^{1,2}(\Omega)$. Assume that $\lambda<\lambda_{1}(f)$ and consider the following problem:

$$
\left\{\begin{array}{l}
-\operatorname{div}\left(|x|^{-(N-2)} \nabla w\right)=\lambda|x|^{-(N-2(\gamma+1)) / 2} f(x)(w+1) \quad \text { in } \Omega, \\
w=0 \text { on } \partial \Omega .
\end{array}\right.
$$

Since $0<\lambda<\lambda_{1}(f)$, the functional

$$
\begin{aligned}
J(v)= & \frac{1}{2} \int_{\Omega}|\nabla w|^{2}|x|^{-(N-2)} d x-\frac{\lambda}{2} \int_{\Omega} f(x) w^{2}|x|^{-(N-2(\gamma+1)) / 2} d x \\
& -\lambda \int_{\Omega} f|x|^{-(N-2(\gamma+1)) / 2} w d x
\end{aligned}
$$

is well defined in $\mathcal{D}_{0,(N-2) / 2}^{1,2}(\Omega)$ and it is easy to check that $J$ is coercive and Fréchetdifferentiable in $\mathcal{D}_{0,(N-2) / 2}^{1,2}(\Omega)$. Therefore by the Ekeland variational principle, there exists a minimizing sequence $\left\{w_{k}\right\}_{k \in \mathbb{N}} \subset \mathcal{D}_{0,(N-2) / 2}^{1,2}(\Omega), w_{k} \geq 0$, such that (i) $w_{k} \rightarrow w$ weakly in $\mathcal{D}_{0,(N-2) / 2}^{1,2}(\Omega)$; (ii) $J\left(w_{k}\right) \rightarrow c=\inf _{\phi \in \mathcal{D}_{0,(N-2) / 2}^{1,2}(\Omega)} J(\phi)$ and (iii) $J^{\prime}\left(w_{k}\right) \rightarrow 0$. Thus the weak limit function $w \in \mathcal{D}_{0,(N-2) / 2}^{1,2}(\Omega)$, is the unique weak solution to problem (17). Moreover, one can prove that the convergence of the sequence is strong in $\mathcal{D}_{0,(N-2) / 2}^{1,2}(\Omega)$. By setting $v=\log (w+1)$, we find that $v \in \mathcal{D}_{0,(N-2) / 2}^{1,2}(\Omega), e^{v}-1 \in$ $\mathcal{D}_{0,(N-2) / 2}^{1,2}(\Omega)$ and

$$
-\operatorname{div}\left(|x|^{-(N-2)} \nabla v\right)=|x|^{-(N-2)}|\nabla v|^{2}+\lambda|x|^{-(N-2(\gamma+1)) / 2} f(x) .
$$

Hence $u(x)=|x|^{-(N-2(\gamma+1)) / 2} v$ satisfies $u \in H_{\gamma}$ and $u$ solves 13 . 
3.3. Regularity and existence of weaker solutions: Relation to elliptic problems with measure data

We begin by proving the following general regularity result.

Theorem 3.5. Assume that $v \in \mathcal{D}_{0,(N-2) / 2}^{1,2}(\Omega)$ is a solution of problem 14 , where $f \in L^{1}(\Omega)$ satisfies $f(x) \geq 0$ a.e. in $\Omega$. Then

$$
e^{\delta|v|}-1 \in \mathcal{D}_{0,(N-2) / 2}^{1,2}(\Omega) \quad \text { for every } \delta<1 / 2 .
$$

Moreover, if $u$ solves $\left[13\right.$, then $e^{\delta|x|^{(N-2(\gamma+1)) / 2} u}-1 \in \mathcal{D}_{0,(N-2) / 2}^{1,2}(\Omega)$ for all $\delta<1 / 2$.

Proof. The assertion follows by using $e^{\delta|v| /(1+\varepsilon|v|)}-1$ as a test function in 14 and by letting $\varepsilon \rightarrow 0$. (See [1] for the details in a simpler but similar setting.)

We define the capacity of a subset $E$ of $\Omega$ with respect to the space $\mathcal{D}_{0,(N-2) / 2}^{1,2}(\Omega)$ by

$$
\operatorname{cap}(E)=\inf \left\{\int_{\Omega}|\nabla \phi|^{2}|x|^{-(N-2)} d x \mid \phi \in \mathcal{D}_{0,(N-2) / 2}^{1,2}(\Omega) \text { and } \phi \geq 1 \text { in } E\right\} .
$$

Theorem 3.6. For a positive function $f \in L^{1}\left(\Omega,|x|^{-(N-2(\gamma+1)) / 2}\right)$, let $u \in H_{\gamma}(\Omega)$ be a solution to problem $(13)$. Set $w=e^{|x|^{(N-2(\gamma+1)) / 2} u}-1$. Then there exists a measure $\mu_{s}$, concentrated on a set of zero capacity, such that

$$
\left\{\begin{array}{l}
-\operatorname{div}\left(|x|^{-(N-2)} \nabla w\right)=\lambda|x|^{-(N-2(\gamma+1)) / 2} f(x)(w+1)+\mu_{s} \quad \text { in } \mathcal{D}^{\prime}(\Omega), \\
w=0 \text { on } \partial \Omega .
\end{array}\right.
$$

Moreover, $\mu_{s}$ is the following weak limit in the space of bounded Radon measures:

$$
\mu_{s}=\lim _{\varepsilon \rightarrow 0}|x|^{-(N-2)}|\nabla v|^{2} e^{v /(1+\varepsilon v)}\left(1-\frac{1}{(1+\varepsilon v)^{2}}\right) .
$$

Proof. Since $\lambda$ does not play any role, we will take $\lambda=1$. As above we set $v(x)=$ $|x|^{(N-2(\gamma+1)) / 2} u(x)$. Then $v$ solves the problem

$$
\left\{\begin{array}{l}
-\operatorname{div}\left(|x|^{-(N-2)} \nabla v\right)=|x|^{-(N-2)}|\nabla v|^{2}+|x|^{-(N-2(\gamma+1)) / 2} f(x) \quad \text { in } \Omega, \\
v=0 \text { on } \partial \Omega,
\end{array}\right.
$$

and $v \in \mathcal{D}_{0,(N-2) / 2}^{1,2}(\Omega)$. Defining $w=e^{v}-1$, by Theorem 3.5 and Hölder's inequality we see that $w \in \mathcal{D}_{0, N-2}^{1, q}(\Omega)$ for all $q<N /(N-1)$. Let $\varepsilon>0$. If we use $e^{v /(1+\varepsilon v)}-1$ $\in L^{\infty}(\Omega) \cap \mathcal{D}_{0,(N-2) / 2}^{1,2}(\Omega)$ as a test function in 21 , it follows that

$$
\begin{aligned}
\int_{\Omega} \frac{|\nabla v|^{2}}{|x|^{N-2}} d x= & \int_{\Omega} \frac{|\nabla v|^{2}}{|x|^{(N-2)}} e^{v /(1+\varepsilon v)}\left(1-\frac{1}{(1+\varepsilon v)^{2}}\right) d x \\
& +\int_{\Omega} \frac{f}{|x|^{(N-2(\gamma+1)) / 2}}\left(e^{v /(1+\varepsilon v)}-1\right) d x
\end{aligned}
$$


Hence

$$
\int_{\Omega} f|x|^{-(N-2(\gamma+1)) / 2}\left(e^{v /(1+\varepsilon v)}-1\right) d x \leq \int_{\Omega}|\nabla v|^{2}|x|^{-(N-2)} d x
$$

and by the monotone convergence theorem we conclude that

$$
\int_{\Omega} \frac{f}{|x|^{(N-2(\gamma+1)) / 2}}\left(e^{v /(1+\varepsilon v)}-1\right) \rightarrow \int_{\Omega} \frac{f}{|x|^{(N-2(\gamma+1)) / 2}} w d x \leq \int_{\Omega} \frac{|\nabla v|^{2}}{|x|^{N-2}} d x<\infty .
$$

Similarly, we obtain

$$
\int_{\Omega}|\nabla v|^{2}|x|^{-(N-2)} e^{v /(1+\varepsilon v)}\left(1-\frac{1}{(1+\varepsilon v)^{2}} d x\right) d x \leq \int_{\Omega}|\nabla v|^{2}|x|^{-(N-2)} d x .
$$

Then, up to a subsequence,

$$
|\nabla v|^{2}|x|^{-(N-2)} e^{v /(1+\varepsilon v)}\left(1-\frac{1}{(1+\varepsilon v)^{2}}\right) \rightarrow \mu_{s}
$$

where $\mu_{s}$ is a positive Radon measure. Notice that $\mu_{s}$ is concentrated on the set $A \equiv$ $\{x \in \Omega: v(x)=\infty\}$ in the sense that

$$
\int_{v \leq k}|\nabla v|^{2} e^{v /(1+\varepsilon v)}\left(1-\frac{1}{(1+\varepsilon v)^{2}}\right)|x|^{-(N-2)} d x \rightarrow 0 \quad \text { as } \varepsilon \rightarrow 0 \text { for all } k>0 .
$$

Since $v \in \mathcal{D}_{0,(N-2) / 2}^{1,2}(\Omega)$, we have $\operatorname{cap}(A)=0$.

Define $w_{\varepsilon}(x)=\int_{0}^{v(x)} e^{s /(1+\varepsilon s)} d s \in \mathcal{D}_{0,(N-2) / 2}^{1,2}(\Omega)$. Then $w_{\varepsilon}$ solves

$$
\begin{aligned}
-\operatorname{div}\left(|x|^{-(N-2)} \nabla w_{\varepsilon}\right)= & e^{v /(1+\varepsilon v)}|\nabla v|^{2}|x|^{-(N-2)}\left(1-\frac{1}{(1+\varepsilon v)^{2}}\right) \\
& +f(x)|x|^{-(N-2(\gamma+1)) / 2} e^{v /(1+\varepsilon v)}
\end{aligned}
$$

in the sense of distributions. The last term converges in $L^{1}(\Omega)$ by (22), while the remaining one converges to $\mu_{s}$. Since $v_{\varepsilon} \rightarrow v$ in $L^{1}(\Omega)$, it follows that $v$ satisfies equation (19) in the sense of distributions. Therefore $\mu_{s}$ is uniquely determined and the convergence in 20] holds for the whole sequence.

Theorem 3.7. Assume $f$ is a positive function such that hypothesis $(\mathbf{B})$ holds. Let $\mu$ be a positive Radon measure with bounded total variation such that $\operatorname{con}(\mu) \subset \Omega \backslash B_{a}(0)$. Then, for all $\lambda<\lambda_{1}(f)$, the problem

$$
\left\{\begin{array}{l}
-\operatorname{div}\left(|x|^{-(N-2)} \nabla w\right)=\lambda f(x)|x|^{-(N-2(\gamma+1)) / 2}(w+1)+\mu \quad \text { in } \mathcal{D}^{\prime}(\Omega), \\
w \in \mathcal{D}_{0, N-2}^{1, q}(\Omega) \quad \text { for all } q<N /(N-1), \\
T_{k}(w) \in \mathcal{D}_{0,(N-2) / 2}^{1,2}(\Omega), \quad \log (1+w) \in \mathcal{D}_{0,(N-2) / 2}^{1,2}(\Omega),
\end{array}\right.
$$

has a minimal weak positive solution $w$. 
The proof is a direct application of the existence Theorem 2.2. The fact that $\log (1+w) \in$ $\mathcal{D}_{0,(N-2) / 2}^{1,2}(\Omega)$ follows by using a similar argument to the proof of Theorem 2.13 in [1]. A sufficient condition for $(\mathbf{B})$ to hold is that $f|x|^{(N+2(\gamma-1)) / 2} \in L^{p}(\Omega), p>N / 2$.

As a consequence we obtain the next result.

Theorem 3.8. Let $\mu_{s}$ be a bounded positive measure which is concentrated on a compact set $E \subset \Omega \backslash B_{a}(0)$ of zero capacity, and let $f$ satisfy (B). For $\lambda<\lambda_{1}(f)$, we define $w$ to be the solution of the problem

$$
\left\{\begin{array}{l}
-\operatorname{div}\left(|x|^{-(N-2)} \nabla w\right)=\lambda f(x)|x|^{-(N-2(\gamma+1)) / 2}(w+1)+\mu \quad \text { in } \mathcal{D}^{\prime}(\Omega), \\
w \in \mathcal{D}_{0, N-2}^{1, q}(\Omega) \quad \text { for all } q<N /(N-1), \\
T_{k}(w) \in \mathcal{D}_{0,(N-2) / 2}^{1,2}(\Omega), \quad \log (1+w) \in \mathcal{D}_{0,(N-2) / 2}^{1,2}(\Omega)
\end{array}\right.
$$

Set $v=\log (w+1)$. Then $v$ satisfies

$$
\left\{\begin{array}{l}
-\operatorname{div}\left(|x|^{-(N-2)} \nabla v\right)=|\nabla v|^{2}|x|^{-(N-2)}+\lambda \frac{f(x)}{|x|^{(N-2(\gamma+1)) / 2}} \quad \text { in } \mathcal{D}^{\prime}(\Omega), \\
v \in \mathcal{D}_{0,(N-2) / 2}^{1,2}(\Omega)
\end{array}\right.
$$

Moreover, $u(x)=|x|^{-(N-2(\gamma+1)) / 2} v$ belongs to $H_{\gamma}$ and is a weak solution to 13 .

Proof. The existence of $w$ and the fact that $v=\log (w+1) \in \mathcal{D}_{0,(N-2) / 2}^{1,2}(\Omega)$ are an application of Theorem 3.7. In order to prove that $v$ satisfies 26, define a sequence $\left\{g_{n}\right\}$ of positive bounded functions such that $\left\|g_{n}\right\|_{1} \leq c$ and $g_{n} \rightarrow \mu_{s}$ in the sense of measures. Let $w_{n}$ be the unique solution to

$$
\left\{\begin{array}{l}
-\operatorname{div}\left(|x|^{-(N-2)} \nabla w_{n}\right)=\lambda T_{n}\left(f|x|^{-(N-2(\gamma+1)) / 2}(w+1)\right)+g_{n}(x) \quad \text { in } \Omega, \\
w_{n} \in \mathcal{D}_{0,(N-2) / 2}^{1,2}(\Omega)
\end{array}\right.
$$

It is easy to check that $w_{n} \rightarrow w$ in $\mathcal{D}_{0, N-2}^{1, q}(\Omega)$ for all $q<N /(N-1)$ (see [2] and [4] for details). We set $v_{n}=\log \left(1+w_{n}\right)$. Then by a direct computation one obtains

$$
-\operatorname{div}\left(|x|^{-(N-2)} \nabla v_{n}\right)=\frac{\left|\nabla v_{n}\right|^{2}}{|x|^{N-2}}+\lambda \frac{T_{n}\left(f(x)|x|^{-(N-2(\gamma+1)) / 2}(w+1)\right)}{w_{n}+1}+\frac{g_{n}}{w_{n}+1}
$$

in $\mathcal{D}^{\prime}(\Omega)$. Notice that the result follows if we prove that the right hand side of (28) converges to $|\nabla v|^{2}|x|^{-(N-2)}+\lambda f|x|^{-(N-2(\gamma+1)) / 2}$ in $\mathcal{D}^{\prime}(\Omega)$. We have

$$
\frac{T_{n}\left(f|x|^{-(N-2(\gamma+1)) / 2}(w+1)\right)}{w_{n}+1} \rightarrow f(x)|x|^{-(N-2(\gamma+1)) / 2} \quad \text { in } L^{1}(\Omega) .
$$

We claim that $g_{n} /\left(w_{n}+1\right) \rightarrow 0$ in $\mathcal{D}^{\prime}(\Omega)$. To prove this, consider $A \subset \Omega$ with $\operatorname{cap}(A)=0$ and $\mu$ concentrated on $A$. Then, by a direct computation, for all $\varepsilon>0$ there exists an open set $U_{\varepsilon}$ such that $A \subset U_{\varepsilon}$ and $\operatorname{cap}\left(U_{\varepsilon}\right) \leq \varepsilon$. Thus, for all $\varepsilon>0$ there exists $\phi \in \mathcal{C}_{0}^{\infty}(\Omega)$ 
such that $\phi \geq 0, \phi \equiv 1$ in $U_{\varepsilon}$ and $\int_{\Omega}|\nabla \phi|^{2}|x|^{-(N-2)} d x \leq 2 \varepsilon$. Using the Picone inequality as in [2], we get

$$
\int_{\Omega}|\nabla \phi|^{2}|x|^{-(N-2)} d x \geq \int_{\Omega} \frac{-\operatorname{div}\left(|x|^{-(N-2)} \nabla\left(w_{n}+1\right)\right)}{w_{n}+1} \phi^{2} d x \geq \int_{U_{\varepsilon}} \frac{g_{n}}{w_{n}+1} d x .
$$

Hence we conclude that

$$
\int_{U_{\varepsilon}} \frac{g_{n}}{w_{n}+1} d x \leq 2 \varepsilon \quad \text { for every } n
$$

Let $\phi \in \mathcal{C}_{0}^{\infty}(\Omega)$. We prove that

$$
\lim _{n \rightarrow \infty} \int_{\Omega} \phi \frac{g_{n}}{w_{n}+1} d x=0
$$

Since

$$
\int_{\Omega} \phi \frac{g_{n}}{w_{n}+1} d x=\int_{U_{\varepsilon}} \phi \frac{g_{n}}{w_{n}+1} d x+\int_{\Omega \backslash U_{\varepsilon}} \phi \frac{g_{n}}{w_{n}+1} d x
$$

we have

$$
\begin{aligned}
\left|\int_{\Omega} \phi \frac{g_{n}}{w_{n}+1} d x\right| & \leq\|\phi\|_{\infty} \int_{U_{\varepsilon}} \frac{g_{n}}{w_{n}+1} d x+\int_{\Omega \backslash U_{\varepsilon}}|\phi| g_{n} d x \\
& \leq 2 \varepsilon\|\phi\|_{\infty}+\int_{\Omega \backslash U_{\varepsilon}}|\phi| g_{n} d x .
\end{aligned}
$$

As $g_{n} \rightarrow \mu_{s}$ in the sense of measures and $\mu$ is concentrated on $A \subset U_{\varepsilon}$, we conclude that $\int_{\Omega \backslash U_{\varepsilon}}|\phi| g_{n} d x \rightarrow 0$ as $n \rightarrow \infty$ and the claim follows. To finish the proof, let us show that $\left|\nabla v_{n}\right|^{2}|x|^{-(N-2)} \rightarrow|\nabla v|^{2}|x|^{-(N-2)}$ strongly in $L^{1}(\Omega)$, that is,

$$
\frac{\left|\nabla w_{n}\right|^{2}}{\left(1+w_{n}\right)^{2}}|x|^{-(N-2)} \rightarrow \frac{|\nabla w|^{2}}{(1+w)^{2}}|x|^{-(N-2)} \quad \text { strongly in } L^{1}(\Omega) .
$$

Since the sequence converges a.e. in $\Omega$, by Vitali's theorem we only have to show that it is equi-integrable. Let $E \subset \Omega$ be a measurable set. For every $\delta \in(0,1)$ and $k>0$, defining $E_{n, k} \equiv E \cap\left\{v_{n} \leq k\right\}$, we obtain

$$
\begin{aligned}
\int_{E} \frac{\left|\nabla w_{n}\right|^{2}}{\left(1+w_{n}\right)^{2}} \frac{d x}{|x|^{N-2}} & =\int_{E_{n, k}} \frac{\left|\nabla w_{n}\right|^{2}}{\left(1+w_{n}\right)^{2}} \frac{d x}{|x|^{N-2}}+\int_{E \backslash E_{n, k}} \frac{\left|\nabla w_{n}\right|^{2}}{\left(1+w_{n}\right)^{2}} \frac{d x}{|x|^{N-2}} \\
& \leq \int_{E}\left|\nabla T_{k}\left(w_{n}\right)\right|^{2} \frac{d x}{|x|^{N-2}}+\frac{1}{(1+k)^{1-\delta}} \int_{\Omega} \frac{\left|\nabla w_{n}\right|^{2}}{\left(1+w_{n}\right)^{1+\delta}} \frac{d x}{|x|^{N-2}} .
\end{aligned}
$$

The last integral is uniformly bounded with respect to $n$, therefore the corresponding term can be made small by choosing $k$ large enough. Moreover, for every $k>0, T_{k}\left(w_{n}\right) \rightarrow$ $T_{k}(w)$ strongly in $\mathcal{D}_{0,(N-2) / 2}^{1,2}(\Omega)$ (see [11]), so the integral $\int_{E}\left|\nabla T_{k}\left(w_{n}\right)\right|^{2}|x|^{-(N-2)} d x$ is uniformly small if the measure of $E$ is small enough. The equi-integrability of the sequence $\left|\nabla v_{n}\right|^{2}|x|^{-(N-2)}$ follows immediately. Hence $v$ solves 26 and then $u \in H_{\gamma}$ solves 13 . 
Acknowledgments. We are grateful to two anonymous referees for their comments about this final version. The second author wishes to thank Professor Haïm Brezis for some useful suggestions about this work.

\section{References}

[1] Abdellaoui, B., Dall'Aglio, A., Peral, I.: Some remarks on elliptic problems with critical growth on the gradient. J. Differential Equations 222, 21-62 (2006) Zbl pre05013584 MR 2200746

[2] Abdellaoui, B., Peral, I.: On quasilinear elliptic equations related to some Caffarelli-KohnNirenberg inequalities. Comm. Pure Appl. Anal. 2, 539-566 (2003) Zbl pre02052060 MR 2019067

[3] Abdellaoui, B., Colorado, E., Peral, I.: Some improved Caffarelli-Kohn-Nirenberg inequalities. Calc. Var. Partial Differential Equations 23, 327-345 (2005) Zbl pre02246503 MR 2142067

[4] Bénilan, P., Boccardo, L., Gallouët, T., Gariepy, R., Pierre, M., Vázquez, J. L.: An $L^{1}$ theory of existence and uniqueness of solutions of nonlinear elliptic equations. Ann. Scuola Norm. Sup. Pisa 22, 241-273 (1995) Zbl 0866.35037 MR 1354907

[5] Berestycki, H., Kamin, S., Sivashinsky, G.: Metastability in a flame front evolution equation. Interfaces Free Bound. 3, 361-392 (2001) Zbl 0991.35097 MR 1869585

[6] Boccardo, L., Murat, F., Puel, J.-P.: Existence de solutions non bornées pour certaines équations quasi-linéaires. Portugal. Math. 41, 507-534 (1982) Zbl 0524.35041 MR 0766873

[7] Boccardo, L., Segura de León, S., Trombetti, C.: Bounded and unbounded solutions for a class of quasi-linear elliptic problems with a quadratic gradient term. J. Math. Pures Appl. 80, 919-940 (2001) Zbl pre01770851 MR 1865381

[8] Brezis, H., Cabré, X.: Some simple nonlinear PDE's without solution. Boll. Un. Mat. Ital. Sez. B (8) 1, 223-262 (1998) Zbl 0907.35048 MR 1638143

[9] Brezis, H., Dupaigne, L., Tesei, A.: On a semilinear equation with inverse-square potential. Selecta Math. 11, 1-7 (2005) MR 2179651

[10] Brezis, H., Vázquez, J. L.: Blow-up solutions of some nonlinear elliptic problems. Rev. Mat. Univ. Complut. Madrid 10, 443-469 (1997) Zbl 0894.35038 MR 1605678

[11] Dal Maso, G., Murat, F., Orsina, L., Prignet, A.: Renormalized solutions of elliptic equations with general measure data. Ann. Scuola Norm. Sup. Pisa 28, 741-808 (1999) Zbl 0958.35045 MR 1760541

[12] Ferone, V., Murat, F.: Nonlinear problems having natural growth in the gradient: an existence result when the source terms are small. Nonlinear Anal. 42, 1309-1326 (2000) Zbl pre01529452 MR 1780731

[13] Hansson, K., Maz'ya, V. G., Verbitsky, I. E.: Criteria of solvability for multidimensional Riccati equations. Ark. Mat. 37, 87-120 (1999) Zbl pre02062427 MR 1673427

[14] Kardar, M., Parisi, G., Zhang, Y. C.: Dynamic scaling of growing interfaces. Phys. Rev. Lett. 56, 889-892 (1986)

[15] Lions, P. L., Generalized Solutions of Hamilton-Jacobi Equations. Res. Notes Math. 69, Pitman (1982) Zbl 0497.35001 MR 0667669

[16] Pinchover, Y., Tintarev, K.: Existence of minimizers for Schrödinger equations under domain perturbations with applications to Hardy's inequality. Indiana Univ. Math. J. 54, 1061-1074 (2005) Zbl pre02228528 MR 2164418

[17] Wang, Z. Q., Willem, M.: Caffarelli-Kohn-Nirenberg inequalities with remainder terms. J. Funct. Anal. 203, 550-568 (2003) Zbl 1037.26014 MR 2003359 